\title{
Die Energie- und Klimapolitik der EU - zwischen Implementierung und strategischer Neuorientierung
}

\author{
Oliver Geden*
}

Seit den ehrgeizigen und weltweit beachteten Beschlüssen beim Europäischen Rat im März 2007 ist die Europäische Union ihrem Anspruch, eine integrierte Energie- und Klimapolitik aufzubauen, noch nicht sehr viel nähergekommen. Zwar wurden bislang 13 einschlägige Gesetzgebungsverfahren eröffnet, ${ }^{1}$ die auch in einer breiteren politischen Öffentlichkeit für erheblichen Diskussionsstoff sorgten, zur Verabschiedung neuer Rechtsakte kam es seither jedoch noch nicht. Allenfalls bei einigen wenigen Dossiers ist mit einer schnellen Einigung zu rechnen. Ob es tatsächlich wie geplant möglich sein wird, die wichtigsten Verfahren noch in der laufenden Legislaturperiode des Europäischen Parlaments zum Abschluss zu bringen, erscheint dementsprechend fraglich.

Aufgrund der Komplexität der Materie und da die laufenden Legislativverfahren stark miteinander verwoben sind, wird sich in den ausstehenden Verhandlungen zwischen Rat und Parlament häufig erst relativ spät erkennen lassen, wo exakt die interinstitutionellen Kompromisslinien liegen - ganz gleich, wie sich die Beteiligten bis dahin öffentlich äußern mögen. Dass sich die Energie- und Klimapolitik der Europäischen Union darüber hinaus nicht nur in Gesetzgebungsverfahren, sondern zugleich auch in einer ganzen Reihe weiterer Formate materialisiert - unter anderem in der Beteiligung an internationalen Klimaverhandlungen und energieaußenpolitischen Dialogen, in wettbewerbsrechtlichen Verfahren gegen Energieversorgungsunternehmen oder der Festsetzung von Energieeffizienzstandards im Komitologieverfahren -, trägt ebenfalls nicht zu einem hohen Maß an Transparenz in diesem Politikfeld bei.

\section{Der Steuerungsrahmen: Energiestrategie und Energieaktionsplan}

Die derzeit in der europäischen Energie- und Klimapolitik zu beobachtende Dynamik rührt nicht nur daher, dass Problemlagen wie der sich bereits vollziehende Klimawandel, steigende Energiepreise oder drohende Versorgungsengpässe weltweit auf der politischen Agenda nach oben gerückt sind. Sie ist auch Ausdruck eines gewaltigen Nachholbedarfs auf europäischer Ebene. Erst im Herbst 2005, beim informellen Gipfel von Hampton Court, ergriffen die damals 25 Staats- und Regierungschefs die Initiative für den Aufbau einer euro-

Dr. Oliver Geden, Forschungsgruppe EU-Integration, Stiftung Wissenschaft und Politik (SWP), Berlin.

1 Diese Zählung lässt zwei energie- und klimapolitisch relevante Legislativverfahren, die bereits vor dem Frühjahrsgipfel 2007 - und damit unabhängig von dessen Beschlüssen - eröffnet wurden, außer Acht. Es handelt sich dabei zum einen um die Verhandlungen über den Einbezug des Luftverkehrs in das Emissionshandelssystem der EU (Europäische Kommission: Vorschlag für eine Richtlinie des Europäischen Parlaments und des Rates zur Änderung der Richtlinie 2003/87/EG zwecks Einbeziehung des Luftverkehrs in das System für den Handel mit Treibhausgasemissionszertifikaten in der Gemeinschaft, KOM(2006) 818, 2006/0304 (COD)), zum anderen um die Beratungen über eine Verringerung der spezifischen Treibhausgasemissionen von fossilen Kraftstoffen (Europäische Kommission: Vorschlag für eine Richtlinie des Europäischen Parlaments und des Rates zur Änderung der Richtlinie 98/70/EG im Hinblick auf die Spezifikationen für Otto-, Diesel- und Gasölkraftstoffe und die Einführung eines Systems zur Überwachung und Verringerung der Treibhausgasemissionen bei der Verwendung von für den Straßenverkehr bestimmten Kraftstoffen, zur Änderung der Richtlinie 1999/ 32/EG des Rates im Hinblick auf die Spezifikationen für von Binnenschiffen gebrauchte Kraftstoffe und zur Aufhebung der Richtlinie 93/12/EWG, KOM(2007) 18, 2007/0019 (COD)). 
päischen Energiepolitik, die sich nicht wie bis dahin in weitgehend unverbundenen Einzelmaßnahmen erschöpft, sondern einen umfassenden Ansatz verfolgt. Im März 2006 legte die Kommission ein Energie-Grünbuch vor, im Januar 2007 den Entwurf einer Energiestrategie samt daraus abgeleitetem Energieaktionsplan für die Jahre 2007-2009. Während die Strategie vom Europäischen Rat kurz darauf unverändert angenommen wurde, nahmen die Staatsund Regierungschefs beim Aktionsplan einige Modifikationen vor. ${ }^{2}$

In der komplexen Architektur der europäischen Energie- und Klimapolitik bilden Energiestrategie und Energieaktionsplan das Fundament. Die 2007 beschlossene Version beschreibt erstmals einen - wenn auch noch fragilen - Grundkonsens über Notwendigkeit und Ziele einer gemeinsamen, genuin europäischen Politik. Bereits 2009 soll eine revidierte Fassung der Rahmenstrategie vom Europäischen Rat angenommen werden, darauf aufbauend zudem auch ein Aktionsplan für die Jahre ab 2010. Dies böte verfahrenstechnisch die Gelegenheit, schon bald einige grundlegende Konstruktionsfehler im Fundament der EU-Energie- und Klimapolitik zu beheben. Fraglich ist jedoch, ob diese Möglichkeit bereits zu diesem relativ frühen Zeitpunkt genutzt werden kann.

Die im März 2007 angenommene Energiestrategie stellt drei Ziele der europäischen Energieversorgung gleichgewichtig ins Zentrum: Nachhaltigkeit, Wettbewerbsfähigkeit und Versorgungssicherheit. Die dabei auf der Hand liegenden Zielkonflikte blendet die Energiestrategie jedoch weitgehend aus. ${ }^{3}$ Eine explizite Prioritätensetzung fehlt dementsprechend. Dies gilt auch für den aus der Strategie abgeleiteten Energieaktionsplan, der lediglich eine Fülle von Einzelmaßnahmen auflistet, ohne erkennbare Schwerpunktsetzung. ${ }^{4}$ Diese Defizite in Strategie und Aktionsplan mussten 2007 jedoch in Kauf genommen werden, um einen schnellen Konsens unter den Mitgliedstaaten zu erreichen, denn deren energiepolitische Präferenzen liegen weit auseinander. ${ }^{5}$ Während in den EU-15 die Bekämpfung und Eindämmung des Klimawandels sehr weit oben auf der Agenda steht, herrscht diesbezüglich in den neuen mittelosteuropäischen Mitgliedstaaten allenfalls freundliches Desinteresse. Hier steht die Gewährleistung von Energieversorgungssicherheit deutlich an erster Stelle, vor allem das Verhältnis zu Russland, dem mit Abstand wichtigsten Öl- und Gaslieferanten der Region. Das Ziel einer wettbewerbsfähigen - sprich: relativ preisgünstigen - Energieversorgung wird im Grundsatz zwar von allen Regierungen befürwortet. Das für eine Steigerung der Wettbewerbsdynamik zentrale Projekt der Schaffung eines funktionierenden europäischen Energiebinnenmarkts wird jedoch nur von denjenigen Mitgliedstaaten mit Nachdruck gefordert, die - wie Großbritannien, die Niederlande oder Schweden - bereits über weitgehend liberalisierte Energiemärkte verfügen. ${ }^{6}$ Die Struktur des ,energiepolitischen Zieldreiecks $^{6}$ - das in ähnlicher Weise auch in der deutschen Energiepolitik Verwendung findet weist dementsprechend einen unbestreitbaren Vorteil auf: Alle an der frühen Aufbauphase einer ,integrierten europäischen Energie- und Klimapolitik' beteiligten Akteure fanden ihre Präferenzen zunächst einmal in angemessener Form repräsentiert. Der auf diese Weise vermiedene und potenziell langwierige Streit über die richtigen Prioritätensetzungen wurde da-

2 Vgl. Oliver Geden/Severin Fischer: Die Energie- und Klimapolitik der Europäischen Union. Bestandsaufnahme und Perspektiven, Baden-Baden 2008, S. 23-30.

3 Vgl. Europäische Kommission: Eine Energiepolitik für Europa, $\operatorname{KOM(2007)~1,~S.~3-6.~}$

4 Vgl. Rat der Europäischen Union: Europäischer Rat Brüssel, 8./9. März 2007. Schlussfolgerungen des Vorsitzes, Dok. 7224/1/07 REV 1, S. 10-23.

$5 \mathrm{Zu}$ den entscheidenden Verhandlungen während der ersten Hälfte der deutschen Ratspräsidentschaft vgl. Susanne Dröge/Oliver Geden: Weitreichende Grundsatzentscheidungen für eine integrierte Energie- und Klimapolitik, in: Daniela Kietz/Volker Perthes (Hrsg): Handlungsspielräume einer EU-Ratspräsidentschaft. Eine Funktionsanalyse des deutschen Vorsitzes im ersten Halbjahr 2007, SWP-Studie 24/2007, S. 48-52.

6 Vgl. Institut für Europäische Politik (Hrsg.): EU-25/27 Watch No. 5, Berlin 2007, S. 78-115. 
mit jedoch unweigerlich in die derzeit laufenden Gesetzgebungsverfahren verlagert, mit dem Effekt, dass deren Verlauf zu Beginn für alle Beteiligten jeweils nur noch in einem sehr eingeschränkten Maße kalkulierbar ist.

Doch auch wenn seitens der beteiligten Akteursgruppen immer wieder betont wird, dass ein Gleichgewicht der drei grundlegenden energiepolitischen Ziele anzustreben sei - auch wenn das jeweilige ,Gleichgewichtsempfinden“ sehr variiert -, so lässt sich in der europäischen Energiepolitik doch eine latente Priorität des Nachhaltigkeitsziels ausmachen. Obgleich in der öffentlichen Debatte wenig beachtet, legt schon die Energiestrategie den Fokus sehr stark auf die internationale Klimapolitik, indem sie die Beschränkung des globalen Temperaturanstiegs auf 2 Grad Celsius (gegenüber dem vorindustriellen Niveau) ausdrücklich als strategisches Ziel der europäischen Energiepolitik deklariert. Alle Bemühungen, dieses strategische Ziel zu erreichen, würden nicht nur die Umweltfreundlichkeit, sondern in der Regel auch die Wirtschaftlichkeit und die Sicherheit der europäischen Energieversorgung befördern. ${ }^{7}$ Betrachtet man zudem auch die nur wenigen quantifizierten Teilziele, für die laut Energiestrategie und Energieaktionsplan ein rechtsverbindlicher Charakter anzustreben ist und die damit die potenziell größte Steuerungswirkung entfalten werden, so bestätigt sich die Dominanz des Nachhaltigkeitsziels. Bis zum Jahr 2020 sollen die Treibhausgasemissionen gegenüber dem Stand von 1990 in jedem Fall um 20 Prozent gesenkt werden, gar um 30 Prozent, wenn es bei den derzeit laufenden Verhandlungen über ein Post-KyotoAbkommen zu einer ehrgeizigen Vereinbarung unter Einbezug aller Industrie- und Schwellenländer käme. Darüber hinaus soll der Anteil der erneuerbaren Energieträger im gleichen Zeitraum auf 20 Prozent des Endenergieverbrauchs gesteigert werden. Da diese Zielmarken bislang auch im Mittelpunkt der Kommunikationsbemühungen der Europäischen Union stehen, werden die europäischen Gesetzgebungsorgane daran keine Abstriche vornehmen können, ohne einen massiven Ansehensverlust zu riskieren, nicht nur gegenüber der europäischen, sondern auch einer globalen Öffentlichkeit.

\section{Dauerbaustelle Energiebinnenmarkt}

Den Auftakt der energiepolitisch relevanten Legislativverfahren bildete das im September 2007 von der Kommission vorgelegte ,Dritte Binnenmarktpaket', das insgesamt fünf Gesetzgebungsvorschläge enthält, die derzeit in Rat und Parlament beraten werden (COD/ 2007/195-199). Hierzu zählen Revisionen der beiden bestehenden Binnenmarktrichtlinien für Strom beziehungsweise Gas, eine Verordnung zur Errichtung einer EU-Agentur für die Koordination der nationalen Energieregulierungsbehörden sowie zwei Verordnungen zur Regulierung grenzüberschreitender Netzverbindungen (,Interkonnektoren') bei Strom beziehungsweise Gas. ${ }^{8}$

7 Vgl. Europäische Kommission: Energiepolitik für Europa, 2007, S. 5-6.

8 Europäische Kommission: Vorschlag für eine Richtlinie des Europäischen Parlaments und des Rates zur Änderung der Richtlinie 2003/54/EG über gemeinsame Vorschriften für den Elektrizitätsbinnenmarkt, KOM(2007) 528, 2007/0195 (COD); Vorschlag für eine Richtlinie des Europäischen Parlaments und des Rates zur Änderung der Richtlinie 2003/55/EG über gemeinsame Vorschriften für den Erdgasbinnenmarkt, KOM(2007) 529, 2007/0196 (COD); Vorschlag für eine Verordnung des Europäischen Parlaments und des Rates zur Gründung einer Agentur für die Zusammenarbeit der Energieregulierungsbehörden, KOM(2007) 530, 2007/0197 (COD); Vorschlag für eine Verordnung des Europäischen Parlaments und des Rates zur Änderung der Verordnung (EG) Nr. 1228/2003 über die Netzzugangsbedingungen für den grenzüberschreitenden Stromhandel, $\operatorname{KOM}(2007)$ 531, 2007/0198 (COD); Vorschlag für eine Verordnung des Europäischen Parlaments und des Rates zur Änderung der Verordnung (EG) Nr. 1775/2005 über die Bedingungen für den Zugang zu den Erdgasfernleitungsnetzen, $\mathrm{KOM}(2007)$ 532, 2007/0199 (COD). 
Während das Ziel einer umweltverträglichen Energieversorgung im Fokus der öffentlichen Aufmerksamkeit steht und quantifizierte Teilziele einer kritischen Evaluierung zudem sehr leicht zugänglich sind, verhält es sich bei der Herausforderung einer wettbewerbsfähigen Energieversorgungsstruktur weitaus komplizierter. Fortschritte sind in diesem Bereich ungleich schwerer zu messen. ${ }^{9}$ Positive Preiseffekte zeigen sich gegenwärtig nur noch als Dämpfung von fast unvermeidlichen Preisanstiegen, für Ausbau und Modernisierung der Erzeugungs- und Transportinfrastruktur existieren keine quantifizierbaren Zielvorgaben. Der Grad der Wettbewerbswirksamkeit neuer Rechtsakte lässt sich aufgrund der Komplexität der europäischen Energiemärkte nur sehr eingeschränkt vorhersagen. Vor allem das für die Erhöhung der Wettbewerbsfähigkeit zentrale Projekt der Schaffung eines funktionierenden Binnenmarkts für die leitungsgebundenen Energieträger Strom und Gas kam in den letzten 15 Jahren nur sehr schleppend voran. Mit Nachdruck wurde die Vollendung des Energiebinnenmarkts seit Mitte der Neunzigerjahre fast ausschließlich von der Kommission betrieben. Viele Mitgliedstaaten lassen sich bis heute primär vom Prinzip der nationalen Souveränität in Fragen der Energieversorgung leiten und schotten ihre Märkte nach außen hin so weit wie möglich ab. Veränderungswille dokumentiert sich bislang häufig nur in den Schlussfolgerungen des Europäischen Rats. Legislativvorschläge der Kommission wurden in der Vergangenheit regelmäßig abgeschwächt oder bei der Umsetzung in nationales Recht mit zahlreichen Schlupflöchern versehen. ${ }^{10}$

Schon seit 2006 zeichnet sich ab, dass die Kommission das zentrale Wettbewerbshindernis in der Existenz, vertikal integrierter ' Energieversorgungsunternehmen sieht. Diese sind nicht nur in der Stromproduktion beziehungsweise in der Förderung oder dem Import von Gas engagiert, sie verfügen auch über das Eigentum an den Übertragungs- und Fernleitungsnetzen. Auf diese Weise entstehen ihnen zahlreiche Möglichkeiten, in- wie ausländische Konkurrenten beim Markteintritt und beim Zugang zu privaten wie gewerblichen Endverbrauchern zu behindern. Die Kommission bevorzugt deshalb in ihren Vorschlägen zur Revision der beiden Richtlinien zum Elektrizitäts- sowie zum Gasbinnenmarkt die Radikallösung der „eigentumsrechtlichen Entflechtung“ (Ownership Unbundling). Als zweitbeste Option sieht sie das Modell des „Independent System Operators“ (ISO), bei dem die integrierten Energiekonzerne ihre Netze zwar nicht verkaufen, aber in unabhängige Betreibergesellschaften überführen müssen. Bei den Beratungen über das Binnenmarktpaket im Rat hat sich die Diskussion zunächst fast völlig auf das Für und Wider einzelner Entflechtungsmodelle verengt, spätestens seit der Vorlage einer Alternativoption durch Deutschland, Frankreich und sechs weitere Mitgliedstaaten (,Dritter Weg') im Januar 2008. Da die Positionen der liberalisierungsfreundlichen und -skeptischen Mitgliedstaaten grundsätzlich relativ weit auseinander liegen, konnte beim Energieministerrat im Juni 2008 lediglich eine informelle Grundsatzeinigung erzielt werden, die auf ein „Optionsmodell“ hinausliefe. Jeder Mitgliedstaat könnte demnach entscheiden, ob er innerhalb seiner Grenzen die eigentumsrechtliche Entflechtung, die Schaffung eines „Independent System Operators“ (ISO) oder die mit dem ,Dritten Weg“ eingebrachte Variante eines „Independent Transmission Operators“ (ITO) vorschreibt. Beim ITO-Modell dürfte die Mehrheit der Vorstandsmitglieder einer Netztochter mehrere Jahre vor und nach ihrer Tätigkeit nicht in anderen Konzerneinheiten des Versorgers beschäftigt werden. Diese Netztöchter wären zudem verpflichtet, sich langfristige

9 Vgl. Jacopo Torriti: Does the Impact Assessment on the ,Third Package' provide the correct economic forecast for the liberalisation of the EU energy markets?, EUI Working Papers RSCAS 14/2008; Jacques J. de Jong: The Third EU Energy Market Package: Are We Singing the Right Song?, CIEP Briefing Paper 8/2008.

10 Vgl. Geden/Fischer: Energie- und Klimapolitik, 2008, S. 27-28, 70-73. 
Investitionspläne von den nationalen Energieregulierungsbehörden genehmigen zu lassen. ${ }^{11}$ Da die ,Dritte Weg '-Gruppe ${ }^{12}$ stark genug ist, um eine Einigung im Rat scheitern zu lassen, dürfte ihre Verhandlungsmacht ausreichen, um das Optionsmodell inklusive der ITO-Variante auch in den kommenden Auseinandersetzungen mit dem Europäischen Parlament durchzusetzen. Das Europäische Parlament hat über alle Teile des Binnenmarktpakets im Juni/Juli 2008 bereits in 1. Lesung abgestimmt, hinsichtlich der Entflechtungsvarianten fielen die Entscheidungen jedoch uneinheitlich aus. Während der ,Dritte Weg ' bei der Abstimmung über die Gasbinnenmarktrichtlinie angenommen wurde, kam es beim Votum über den Richtlinienentwurf zum Strombinnenmarkt zur Ablehnung dieser Variante.

Bei näherer Betrachtung der Debatten und Verhandlungen über das Binnenmarktpaket ist festzustellen, dass die zu beobachtende Fokussierung auf die einzelnen Entflechtungsvarianten fast schon ideologische Züge trägt. Kommission und liberalisierungsfreundliche Mitgliedstaaten, Parlamentarier und Interessengruppen erwecken den Eindruck, das Ownership Unbundling bedeute die Lösung aller Probleme im Energiebinnenmarkt, die Gegner der eigentumsrechtlichen Entflechtung malen beständig Schreckensszenarien an die Wand und warnen vor einer ,Zerschlagung ' nationaler oder europäischer Energie-Champions. Der Mittelweg, der bei den beiden Binnenmarktrichtlinien voraussichtlich eingeschlagen werden wird, kann durchaus als weiterer Schritt in die richtige Richtung gewertet werden - und fügt sich damit nahtlos in die Geschichte der Bemühungen um die Liberalisierung der europäischen Strom- und Gasmärkte ein. Eine auf Betreiben der Liberalisierungsbefürworter eingefügte Klausel, nach der die Kommission die Wettbewerbseffekte der revidierten Binnenmarktrichtlinien zwei bis drei Jahre nach ihrer Umsetzung erneut prüfen und wenn notwendig weitergehende Legislativvorschläge unterbreiten soll, wird wohl schon in wenigen Jahren zur Vorlage neuer Initiativen führen. ${ }^{13}$

Im Schatten der Entflechtungsdiskussionen hat das Parlament in den 1. Lesungen der fünf Legislativvorschläge des Binnenmarktpakets in zahlreichen Punkten (zum Teil gravierende) Verschärfungen der ursprünglichen Kommissionsvorschläge vorgenommen. Dies betrifft nicht nur die Ausweitung der Rechte privater Endverbraucher, sondern auch die deutliche Erweiterung der Kompetenzen der nationalen Regulierungsbehörden sowie die Ausstattung einer europäischen Regulierungsagentur mit eigenständigen Befugnissen. Da die Mitgliedstaaten die Ausweitung der gemeinschaftlichen Regulierungskompetenzen bislang strikt ablehnen, dürfte hier im Laufe der interinstitutionellen Verhandlungen eines der größten Konfliktfelder entstehen. Zudem sind aufgrund der bisherigen Fokussierung auf die Entflechtungsoptionen viele wichtige Details des Binnenmarktpakets bislang noch kaum ernsthaft verhandelt worden, etwa die für die Schaffung eines funktionierenden Energiebinnenmarkts zentralen Regulierungsvorgaben für die grenzüberschreitenden Strom- und Gasnetze. Es kann deshalb noch kaum vorhergesagt werden, bis wann die fünf Gesetzgebungsverfahren zum Abschluss gebracht sein werden, zumal Frankreich erklärt hat, es wolle den Verhandlungen über das Binnenmarktpaket während seiner Ratspräsidentschaft keine hohe Priorität einräumen - zugunsten der laufenden Verfahren mit primär klimapolitischem Bezug.

11 Vgl. Rat der Europäischen Union: Tagung des Rates (Verkehr, Telekommunikation und Energie) am 6. Juni 2008 - Energiebinnenmarkt, Dok. 10513/08.

12 Ihr gehören neben Deutschland und Frankreich auch Österreich, Griechenland, Luxemburg, Lettland, Bulgarien und die Slowakei an.

13 Daneben dürfte die Kommission auch in Zukunft an der Praxis festhalten, integrierte Energieversorger im Rahmen von Kartellrechtsverfahren dazu zu bewegen, ihre Netze zu veräußern, um empfindlichen Geldbußen zu entgehen. Entsprechende Vereinbarungen konnte sie im Februar und Mai 2008 mit zwei deutschen Unternehmen abschließen. E.ON verpflichtete sich dabei zum Verkauf seines Stromübertragungsnetzes, RWE zur Veräußerung seines Gasfernleitungsnetzes. 


\section{Vorleistungen auf dem Weg nach Kopenhagen}

Die starke Verschränkung interner und externer Politiken ist derzeit eines der prägenden Strukturierungsprinzipien der europäischen Energie- und Klimapolitik. Allerdings wirkt die externe Dimension - entsprechend der strategischen Orientierung an dem nur in internationalen Klimaverhandlungen zu erreichenden 2 Grad-Ziel - weitaus stärker auf die interne Dimension als umgekehrt. Die Selbstverpflichtung auf eine unilaterale Reduktion der Treibhausgasemissionen um mindestens 20 Prozent war ausdrücklich auch dazu gedacht, die festgefahrenen Gespräche über ein ehrgeiziges Post-Kyoto-Abkommen wieder in Gang zu bringen. Ermutigt durch die Ergebnisse des G8-Gipfels in Heiligendamm im Juni 2007 gewann bei den EU-Organen die Haltung Oberhand, zunächst möglichst alles zu vermeiden, was die internationalen Verhandlungen (sowie die eigene Vorreiterrolle darin) in negativer Weise beeinträchtigen könnte. ${ }^{14}$ Ausdruck findet dies nicht zuletzt in verfahrenstechnischen Fragen. So verschob die Kommission im Spätherbst 2007 die Eröffnung des Gesetzgebungsverfahrens für eine Verordnung über $\mathrm{CO}_{2}$-Emissionsnormen bei $\mathrm{Pkws}^{15}$ sowie die Vorlage des kompletten Energie- und Klimapakets ${ }^{16}$ bewusst um mehrere Wochen in die Zukunft, um zunächst das Ende der UN-Klimakonferenz auf Bali abzuwarten. Hatte man ursprünglich gehofft, mit einer Vorlage noch vor Bali international Handlungsfähigkeit beweisen zu können, so setzte sich bald die Befürchtung durch, der zu erwartende Streit der Mitgliedstaaten über diese Gesetzesvorschläge würde das Ansehen der Europäischen Union beschädigen. Von ähnlichen Annahmen werden auch die Verhandlungen zwischen Rat und Parlament geprägt sein. Alle klimapolitisch relevanten Rechtsakte sollen nach Ansicht der EUOrgane rechtzeitig vor der (vor-)entscheidenden UN-Klimakonferenz in Kopenhagen im Dezember 2009 verabschiedet werden, möglichst noch vor Ende der laufenden Legislaturperiode des Parlaments. Die Europäische Union würde einen massiven Ansehensverlust riskieren, wenn sie den ehrgeizigen Ankündigungen des Frühjahrsgipfels 2007 nicht auch angemessene Taten folgen ließe. Dementsprechend stehen die Verhandlungen unter einem enormen Zeit- und Erfolgsdruck. Die 1. Lesungen im Parlament werden bis Dezember 2008 abgeschlossen sein, schnelle first reading agreements mit dem Rat dürften jedoch die Ausnahme bleiben. Ohnehin wird es im Rat recht schwierig werden, die Konsensfindung zwi-

14 Vgl. Sebastian Oberthür/Claire Roche Kelly: EU Leadership in International Climate Policy: Achievements and Challenges, in: The International Spectator 3/2008, S. 35-50.

15 Europäische Kommission: Vorschlag für eine Verordnung des Europäischen Parlaments und des Rates zur Festsetzung von Emissionsnormen für neue Personenkraftwagen im Rahmen des Gesamtkonzepts der Gemeinschaft zur Verringerung der CO2-Emissionen von Personenkraftwagen und leichten Nutzfahrzeugen, $\operatorname{KOM}(2007)$ 856, 2007/0297 (COD).

16 Das im Januar 2008 vorgelegte Paket enthält vier Legislativvorschläge. Diese befassen sich mit der Neugestaltung des Emissionshandelssystems (ETS) ab 2013, mit der Aufteilung der nicht ETS-gebundenen Reduktionsverpflichtungen auf die Mitgliedstaaten, der geologischen Speicherung von $\mathrm{CO}_{2}$ sowie dem Ausbau der erneuerbaren Energien: Europäische Kommission: Vorschlag für eine Richtlinie des Europäischen Parlaments und des Rates zur Änderung der Richtlinie 2003/87/EG zwecks Verbesserung und Ausweitung des EU Systems für den Handel mit Treibhausgasemissionszertifikaten, KOM(2008) 16, 2008/0013 (COD); Vorschlag für eine Entscheidung des Europäischen Parlaments und des Rates über die Anstrengungen der Mitgliedstaaten zur Reduktion ihrer Treibhausgasemissionen mit Blick auf die Erfüllung der Verpflichtungen der Gemeinschaft zur Reduktion der Treibhausgasemissionen bis 2020, $\operatorname{KOM}(2008)$ 17, 2008/0014 (COD); Vorschlag für eine Richtlinie des Europäischen Parlaments und des Rates über die geologische Speicherung von Kohlendioxid und zur Änderung der Richtlinien 85/337/EWG und 96/61/EG des Rates sowie der Richtlinien 2000/60/EG, 2001/80/EG, 2004/35/EG, 2006/12/EG und der Verordnung (EG) Nr. 1013/2006, KOM(2008) 18, 2008/0015 (COD); Vorschlag für eine Richtlinie des Europäischen Parlaments und des Rates zur Förderung der Nutzung von Energie aus erneuerbaren Quellen, KOM(2008) 19, 2008/0016 (COD). 
schen den zum Teil sehr unterschiedlichen Interessen der Mitgliedstaaten zu beschleunigen. ${ }^{17}$

Innerhalb des Energie- und Klimapakets richtet sich ein besonderes Augenmerk auf die abermalige Revision des Handelssystems für Emissionszertifikate (ETS), dem seit 2005 zentralen klimapolitischen Instrument der Europäischen Union. Der Richtlinienentwurf der Kommission sieht vor, dass in der Dritten Handelsperiode (2013-2020) der größte Teil der Zertifikate versteigert wird, statt wie bisher weitgehend kostenlos verteilt zu werden. Während Stromproduzenten bereits von 2013 an alle notwendigen Zertifikate zukaufen müssten, könnten energieintensive Industriezweige (Stahl, Aluminium, Zement) zunächst mit Ausnahmeregeln rechnen, die grundsätzlich jedoch bis zum Ende der Handelsperiode auslaufen sollen. Besondere Schutzmechanismen sind für solche Branchen vorgesehen, die weitreichende internationale Wettbewerbsnachteile erleiden würden, falls es im Rahmen eines Post-Kyoto-Abkommens nicht gelänge, Staaten wie China, Indien oder die USA zu signifikanten Emissionsbegrenzungen zu verpflichten. Damit soll auch verhindert werden, dass energieintensive Industriezweige aus der Europäischen Union abwandern, ohne dass deshalb die weltweite Gesamtemissionsmenge sinken würde. Diskutiert wird in der Europäischen Union deshalb etwa das Erheben von Einfuhrzöllen auf Waren aus Ländern, die sich einem internationalen Klimaschutzregime verweigern. Für eine solche Lösung setzt sich insbesondere Frankreich ein. Da solche ,Klimazölle“ aber kaum in Übereinstimmung mit den Regeln der Welthandelsorganisation (WTO) zu bringen sein dürften, bliebe es für energieintensive Branchen - wie derzeit vor allem von Deutschland vorgeschlagen - wohl zunächst bei einer kostenlosen Zuteilung von Emissionszertifikaten. Da die Kommission die internationalen Klimaverhandlungen nicht durch ein gesetzgeberisch bereits vorweggenommenes Scheitern belasten will, sieht ihr Richtlinienentwurf vor, dass über die Definition der betreffenden Industriebranchen sowie die anzustrebenden Maßnahmen erst nach der Weltklimakonferenz in Kopenhagen entschieden wird. Gegen eine solche späte Festlegung opponieren zahlreiche Mitgliedstaaten mit dem Argument, dass die potenziell betroffenen Unternehmen ein Mindestmaß an Planungssicherheit benötigen und deshalb nicht bis 2010 im Ungewissen darüber gelassen werden dürften, ob beziehungsweise welche Ausnahmeregeln für sie ab 2013 in Kraft treten. ${ }^{18}$

Der zweite wesentliche Bruch mit dem bisherigen System läge in der Festlegung einer EU-weiten Emissionsobergrenze. Anders als in den ersten beiden Handelsperioden würde das ETS ab 2013 auf eine Berücksichtigung der volkswirtschaftlichen Ausgangsbedingungen der einzelnen Mitgliedstaaten weitgehend verzichten. Der Kommissionsentwurf sieht zwar die Einrichtung eines Solidaritätsfonds vor, mit dem ein Teil der Auktionierungserlöse umverteilt werden soll, dieser wird jedoch von vielen EU-15-Staaten - darunter Deutschland - abgelehnt. Eine EU-weite Obergrenze würde nicht nur zu einer dringend notwendigen Harmonisierung der Zuteilungskriterien für Zertifikate führen, sondern auch zu einer weiteren Europäisierung der energiepolitischen Handlungsorientierungen. Die Auseinandersetzungen über die bisherige Praxis der Genehmigung Nationaler Allokationspläne durch die Kommission nahm in fast allen Mitgliedstaaten die Form einer Verteidigung nationaler Interessen gegen die ,Zumutungen aus Brüssel' an. Schon gegen die Zuteilungspläne für die Zweite Handelsperiode (2008-2012) haben mehrere mittelosteuropäische Staaten vor dem

17 Vgl. Jennifer Rankin: Cracks appear in climate consensus. East-west split over carbon targets; member states fear effect on businesses, in: European Voice, 12.06.2008.

18 Vgl. Susanne Dröge: ,Klimazölle “ und die Glaubwürdigkeit der EU-Klimastrategie. Internationale Klimapolitik und die Verlagerung von Emissionen (carbon leakage), SWP-Aktuell 72/2008. 
Europäischen Gerichtshof Klage gegen die Kommission erhoben, weil sie sich in ihrer ökonomischen Entwicklung über Gebühr beeinträchtigt sehen, wenn sie an den gleichen Kriterien gemessen werden wie die alten Mitgliedstaaten. Mit dem gleichen Argument wenden sie sich nun auch gegen die EU-weit harmonisierte Ausgabe von Emissionsrechten, werden sich in dieser Frage aber wohl nicht durchsetzen können.

Für die nicht vom ETS erfassten Sektoren (Privathaushalte, Straßenverkehr, Landwirtschaft, Gewerbe), die derzeit für knapp 60 Prozent der europäischen Treibhausgasemissionen verantwortlich sind, soll es nach den Plänen der Kommission beim Prinzip mitgliedstaatlich differenzierter Zielvorgaben (effort sharing) bleiben, einer Methode, die grundsätzlich auch beim Ausbau der erneuerbaren Energieträger angewandt werden wird. Maßgeblich ist hier insbesondere das Bruttoinlandsprodukt pro Kopf. Ausgehend vom neuen Basisjahr 2005 soll es einigen mittelosteuropäischen Staaten erlaubt sein, ihre nicht vom ETS abgedeckten Emissionen bis 2020 noch einmal um bis zu 20 Prozent auszuweiten, während die alten EU-Mitglieder Reduktionen von bis zu 20 Prozent vornehmen sollen. Es kann nicht verwundern, dass sich eine Vielzahl von Mitgliedstaaten durch die von der Kommission vorgeschlagenen Verteilungsschlüssel benachteiligt sieht und bestrebt ist, im Gesetzgebungsverfahren noch vorteilhafte Anpassungen zu erreichen.

Nicht sehr viel anders verhält es sich auch in den Verhandlungen über die Verordnung zur Begrenzung der $\mathrm{CO}_{2}$-Emissionen bei Pkws, ${ }^{19}$ bei denen die Mitgliedstaaten darauf bedacht sind, dass die in ihrem Land ansässige Automobilindustrie keine relativen Wettbewerbsnachteile erleidet. Eine gesetzliche Regelung ist erforderlich geworden, weil die europäischen Autohersteller ihrer früheren Selbstverpflichtung zu deutlichen Emissionsreduktionen bislang nicht nachgekommen sind. Deutschland bestreitet zwar nicht die Notwendigkeit einer gesetzlichen Regelung, erkennt im Kommissions-Vorschlag jedoch eine unzulässige Bevorzugung der französischen und italienischen Kleinwagenproduzenten. ${ }^{20}$ Für den Fall, dass sich ein zwischen Deutschland und Frankreich unter großen Mühen erzielter Kompromissvorschlag nicht durchsetzen lassen sollte - Italien, das sich seit der Amtsübernahme der Regierung Berlusconi hinsichtlich einer nachhaltigen Energiepolitik eher skeptisch äußert, hat bereits deutliche Vorbehalte angemeldet -, ist es nicht ausgeschlossen, dass es im Rat zu einer andauernden Entscheidungsblockade kommt. Der Vorreiterrolle der Europäischen Union bei den UN-Klimaverhandlungen wäre dies jedoch nur bedingt abträglich. Denn auch wenn sich die Mitgliedstaaten im spezifischen Fall der Automobilindustrie nicht auf eine Aufteilung der Belastungen einigen könnten, so würde dies eine Verabschiedung der neuen ETS-Richtlinie und der Entscheidung zum mitgliedstaatlichen effort sharing in den Nicht-ETS-Sektoren vorausgesetzt - dennoch nichts an der geplanten Absenkung der EU-Gesamtemissionen um 20 Prozent ändern. Sollte der PkwVerkehr dazu nur einen als unzulänglich betrachteten Beitrag leisten, so müsste dies an anderer Stelle ausgeglichen werden.

\section{Stiefkind Versorgungssicherheit}

Im dritten Zielkorridor europäischer Energiepolitik, der Gewährleistung von Versorgungssicherheit, sind bislang mit Abstand die geringsten Fortschritte zu verzeichnen. Insbesondere das Vorhaben einer gemeinsamen Energieaußenpolitik kommt kaum über bloße Ab-

19 Europäische Kommission: Vorschlag für eine Verordnung zur Festsetzung von Emissionsnormen für neue Personenkraftwagen, 2007.

20 Vgl. Neue Zürcher Zeitung: „Brüssel“ schreckt die Automobilindustrie auf. Anreize und Strafen zur Reduktion des Kohlendioxid-Ausstosses, 20.12.2007. 
sichtserklärungen hinaus. Der Europäischen Union ist es bisher nicht gelungen, das immer wieder betonte Prinzip des ,Sprechens mit einer Stimme ‘ im Umgang mit Lieferländern von Öl und Gas auch praktisch umzusetzen. Sichtbarstes Zeichen dafür sind die Widersprüche zwischen bilateralen Projekten und gemeinschaftlich beschlossenen Prioritätensetzungen. Aktuellstes Beispiel ist die South Stream-Pipeline, mit der sich Süd- und Mitteleuropa den Zufluss von russischem Gas sichern, zugleich aber die mittelfristige Realisierung des europäischen Vorzeigeprojekts Nabucco gefährden, mit dem Gas vom Kaspischen Meer unter Umgehung von Russland bis nach Österreich geleitet werden soll. Zwar ist das Verhalten der in diesem Zusammenhang sehr häufig kritisierten ungarischen Regierung nachvollziehbar, da die Energieerzeugung Ungarns überdurchschnittlich stark auf Gas basiert, die Lieferzusagen für die Nabucco-Pipeline nach wie vor jedoch nur mangelhaft bleiben. Es ist aber bezeichnend für den geringen Verbindlichkeitsgrad der EU-Energieaußenpolitik, dass die Bekanntgabe entsprechender Vereinbarungen mit Gazprom nur wenige Wochen nach dem Konsensbeschluss über den Energieaktionsplan 2007-2009 erfolgte, bei dem Nabucco zum wichtigsten Gaspipeline-Projekt der Europäischen Union erklärt wurde. ${ }^{21}$ Dieser Widerspruch zwischen gemeinschaftlichen Beschlüssen und nationalstaatlichem Handeln tritt jedoch auch unter umgekehrten Vorzeichen zutage. Obwohl etwa die North Stream-Pipeline von Russland nach Deutschland in den geltenden Leitlinien zu Transeuropäischen Energienetzen als ,,vorrangiges Vorhaben“ eingestuft wird, ${ }^{22}$ sind einige Ostseeanrainerstaaten offenkundig bestrebt, eine Realisierung zumindest zu verzögern.

Dass in der Energieaußenpolitik nach wie vor eine nationalstaatliche Perspektive dominiert, kann angesichts der großen Differenzen zwischen den mitgliedstaatlichen Energieversorgungsstrukturen jedoch kaum überraschen. Die 27 nationalen Energiemärkte unterscheiden sich nicht nur hinsichtlich ihres Energieträgermix deutlich voneinander, sondern auch in Bezug auf den jeweiligen Grad an Importabhängigkeit sowie die Herkunft der Rohstofflieferungen. Dementsprechend haben etwa Spanien und Portugal völlig andere Interessen als etwa Polen und die baltischen Staaten. Eine Annäherung der energieaußenpolitischen Orientierungen ist erst dann realistisch, wenn die externe Energiesicherheitspolitik auch von einer internen Dimension gestützt wird. Grundvoraussetzung dafür ist primär die Schaffung eines funktionierenden Energiebinnenmarkts, der die Übertragungskapazitäten zwischen den mitgliedstaatlichen Netzen erhöhen und damit automatisch auch die Diversifizierungsquoten in den Mitgliedstaaten steigern würde. Insbesondere im Gassektor müssen zukünftig jedoch auch Bevorratungspflichten und Krisenreaktionsmechanismen diskutiert werden. Der Grundstein zur Etablierung von Maßnahmen zur Erhöhung der energiepolitischen Solidarität unter den Mitgliedstaaten wurde mit dem Vertrag von Lissabon gelegt, der - in Art. 122 und Art. 194 des Vertrags über die Arbeitsweise der Europäischen Union - erstmals explizit Klauseln zur Energiesolidarität enthält. Dennoch sollte das Potenzial einer gemeinsamen Energieaußenpolitik keineswegs überschätzt werden. Die in diesem Zusammenhang vielfach geäußerten Hoffnungen auf die mannigfaltigen Effekte eines ,Sprechens mit einer Stimme" sind stark übertrieben. Zum einen stehen die Interessen der eigentlichen Marktakteure, der europäischen Energieimportunternehmen, nicht immer im Einklang mit denen der europäischen Politik. Zum anderen ist die Verhandlungsmacht der Europäischen Union gegenüber Lieferländern beim gegenwärtigen Preisniveau nur sehr begrenzt, was sich auch bei

21 Vgl. Rat der Europäischen Union: Schlussfolgerungen, 2007, S. 18.

22 Entscheidung Nr. 1364/2006/EG des Europäischen Parlaments und des Rates vom 6. September 2006 zur Festlegung von Leitlinien für die transeuropäischen Energienetze und zur Aufhebung der Entscheidung 96/391/EG und der Entscheidung Nr. 1229/2003/EG, in: Amtsblatt der EU, Nr. L 262 vom 22. September 2006, S. 1-23, hier S. 18. 
den laufenden Verhandlungen über energiepolitische Aspekte im neuen Partnerschafts- und Kooperationsabkommen zwischen der Europäischen Union und Russland zeigen wird. ${ }^{23}$

\section{Black Box Energieeffizienz}

In der energiepolitischen Kommunikation der Europäischen Union spielt auch die Steigerung der Energieeffizienz eine Hauptrolle. Dies findet bislang jedoch keine adäquate Entsprechung in der energiepolitischen Praxis - und dass obwohl kein anderer Maßnahmenbereich so sehr geeignet wäre, gleichermaßen zu Umweltverträglichkeit, Wettbewerbsfähigkeit und Sicherheit der europäischen Energieversorgung beizutragen. Hinsichtlich der Zielbestimmung bleibt schon der Energieaktionsplan recht vage. Er postuliert, dass „die Energieeffizienz in der EU erhöht werden muss, damit [...] das Ziel, 20 Prozent des EUEnergieverbrauchs gemessen an den Prognosen für 2020 einzusparen, erreicht wird“ ${ }^{24}$ gibt aber zugleich keinerlei verlässlichen Hinweis darauf, welche Prognosen die Berechnungsgrundlage für das Einsparziel bilden könnten. Dies bedeutet zum einen, dass - anders als von der Europäischen Union permanent kommuniziert - vom Europäischen Rat keineswegs eine 20-prozentige Reduktion des gegenwärtigen Energieverbrauchs vereinbart worden ist, sondern lediglich eine entsprechende Reduktion des für 2020 ursprünglich prognostizierten Verbrauchs. Zum anderen lässt der Energieaktionsplan - nicht anders als alle seither veröffentlichten Dokumente zum Thema - im Dunkeln, wie das maßgebliche Einsparziel konkret lautet oder wie es zu errechnen wäre. ${ }^{25}$

Es passt dementsprechend ins Bild, dass darauf verzichtet wird, eine sanktionierbare Zielmarke für die EU-weite Steigerung der Energieeffizienz festzulegen. Anders als die Senkung der Treibhausgasemissionen um 20 Prozent oder der Ausbau der erneuerbaren auf 20 Prozent soll die angestrebte Senkung des Energieverbrauchs nicht verbindlich sein, der - bis heute ohnehin unbekannte - Zielwert hat lediglich indikativen Charakter. Der im Energieaktionsplan vorgenommene Verweis auf die laufenden Bemühungen im Rahmen gültiger Energieeffizienzrichtlinien ist schon deshalb unbefriedigend, weil die Mitgliedstaaten der gemeinschaftlichen Energieeffizienzpolitik bisher nur einen geringen Stellenwert eingeräumt haben. Bei der Ende 2007/Anfang 2008 erfolgten Evaluierung der nationalen Effizienzaktionspläne monierte die Kommission, dass die Planungen vieler Mitgliedstaaten zwar durchaus ambitioniert ausfielen, von einer adäquaten Umsetzung in die Praxis zumeist aber noch wenig zu sehen sei. ${ }^{26}$ Europäische Initiativen zur Steigerung der Energieeffizienz verlaufen bislang nur sehr schleppend, zum Teil adressieren sie auch nur relativ nachrangige Verbrauchssektoren. So eröffnete die Kommission im Juli 2008 - nach mehrmaliger Verschiebung - zwar drei Gesetzgebungsverfahren im Bereich Energieeffizienz und Umwelt-

23 Vgl. auch Andreas Goldthau/Oliver Geden: Europas Energieversorgungssicherheit - Ein Plädoyer für einen pragmatischen Ansatz, in: Internationale Politik und Gesellschaft 4/2007, S. 58-73.

24 Rat der Europäischen Union: Schlussfolgerungen, 2007, S. 20.

25 Da bisherige Prognosen von einem weiteren Anstieg des Energieverbrauchs in der EU-27 ausgingen, hat die Wahl des Basisjahres 2020 zur Folge, dass der gegenüber dem gegenwärtigen Istzustand zu erreichende Einsparwert unter 20 Prozent liegen wird. Legt man die zum Zeitpunkt der Veröffentlichung des Energieaktionsplans gültige Version des regelmäßig fortgeschriebenen Baseline-Szenarios der Generaldirektion Energie und Verkehr (DG TREN) zugrunde, so beträgt die Differenz zwischen der um 20 Prozent reduzierten Prognose für 2020 (vgl. Europäische Kommission: European Energy and Transport. Trends to 2030 - update 2005, Luxemburg 2006, S. 46) und dem von EUROSTAT für das Jahr 2006 ermittelten realen Primärenergieverbrauch nur 15,8 Prozent.

26 Europäische Kommission: Memo zur ersten Bewertung nationaler Aktionspläne für Energieeffizienz, MEMO/08/32. 
management, die Reichweite der vorgelegten Vorschläge fällt jedoch relativ gering aus. ${ }^{27}$ Die darüber hinaus im Rahmen des Komitologieverfahrens geplante Einführung von verbindlichen Effizienzstandards für einzelne Produktkategorien wie Glühlampen oder den Stand-by-Betrieb bei elektronischen Geräten sind zwar durchaus ein Schritt in die richtige Richtung. Da aber die Stromproduktion ohnehin schon über das Emissionshandelssystem reguliert wird, wäre es vergleichsweise wichtiger, zusätzliche Effizienzanreize in den Sektoren Verkehr und Wärme zu setzen. ${ }^{28}$

\section{Strategic Energy Review - Steuerungsinstrument oder Mittel der politischen Kom- munikation?}

Der Anspruch der Europäischen Union, eine strategiegeleitete Energie- und Klimapolitik zu verfolgen, aber auch die sich bereits abzeichnenden Probleme bei der Implementierung des Energieaktionsplans 2007-2009 machen es notwendig, dass die Europäische Union ihre Steuerungsinstrumente einer selbstkritischen Überprüfung unterzieht. Als zentrales Prozessinstrument ist dafür eine regelmäßige Strategic Energy Review vorgesehen, mit der alle zwei Jahre sowohl eine Modifizierung der eigentlichen Energiestrategie als auch eine Neufassung des Energieaktionsplans vorgenommen werden soll. Die ursprünglich für Anfang 2009 vorgesehene Veröffentlichung der entsprechenden Kommissions-Mitteilung soll auf Wunsch der französischen Ratspräsidentschaft auf den Spätherbst 2008 vorgezogen werden. Die Annahme der Strategic Review ist für den Frühjahrsgipfel 2009 geplant. Eine ernsthafte Debatte über die Angemessenheit der zentralen Ziele und den Erfolg der politischen Instrumente ist zu diesem frühen Zeitpunkt jedoch noch nicht zu erwarten. Es wird vielmehr zu einer Fortschreibung der bisherigen Architektur kommen, auch deutlichere Prioritätensetzungen sind nicht zu erwarten. Dennoch ist davon auszugehen, dass die Review die öffentliche Debatte bis zum Frühjahrsgipfel 2009 anregen wird. Dies nicht nur, weil sie in den Medien und den Mitgliedstaaten einen Raum für kritische Fragen an die bisherigen Fortschritte der EU-Energie- und Klimapolitik eröffnen wird, sondern auch, weil parallel zur Review auch die Vorlage eines Versorgungssicherheits-Pakets geplant ist.

Die Präsentation eines solchen Pakets, das vor allem Instrumente zur Erhöhung der internen Versorgungssicherheit enthalten wird, soll nicht zuletzt signalisieren, dass die Europäische Union sich nicht nur in der Klima- und der Binnenmarktpolitik engagiert, sondern nun auch den dritten Pfeiler ihrer Energiepolitik stärker berücksichtigt. Es ist deshalb zu erwarten, dass Aspekten der Versorgungssicherheit zukünftig etwas mehr Gewicht in der innereuropäischen Debatte zukommen wird, nicht nur im Verhältnis der drei Pfeiler zueinander, sondern als Querschnittsperspektive auch bei der Bewertung von einzelnen Maßnahmen, etwa hinsichtlich der Förderung von Biokraftstoffen, der Zukunft der Atomenergie oder beim Ausbau von Strom- und Gasnetzen. Mit der Stärkung des Pfeilers Versorgungssicher-

27 Es handelt sich dabei um Vorschläge für eine Erweiterung der Ökodesign-Richtlinie (Europäische Kommission: Vorschlag für eine Richtlinie des Europäischen Parlaments und des Rates zur Schaffung eines Rahmens für die Festlegung von Anforderungen an die umweltgerechte Gestaltung von energieverbrauchsrelevanten Produkten, KOM(2008) 399, 2008/0151 (COD)), für eine Verordnung zur Neuregelung des europäischen Umweltzeichens (Europäische Kommission: Vorschlag für eine Verordnung des Europäischen Parlaments und des Rates über eine Regelung für das Umweltzeichen der Gemeinschaft, KOM(2008) 401, 2008/0152 (COD)) sowie für eine Verordnung zur Modifikation des Gemeinschaftssystems für das Umweltmanagement und die Umweltbetriebsprüfung (Europäische Kommission: Vorschlag für eine Verordnung des Europäischen Parlaments und des Rates über die freiwillige Teilnahme von Organisationen an einem Gemeinschaftssystem für Umweltmanagement und Umweltbetriebsprüfung (EMAS), KOM(2008) 402, 2008/0154 (COD)).

28 Vgl. Bodo Sturm: Das Glühbirnenverbot bringt nichts, in: Süddeutsche Zeitung, 19.07.2008. 
heit wird jedoch keine entsprechende Schwächung der Klimaschutz- und Binnenmarktziele einhergehen. Es ist vielmehr zu erwarten, dass die Zahl der - zum Teil widersprüchlichen Ankündigungen und das Ausmaß der letztlich nur schwer einzulösenden Versprechen in der Energie- und Klimapolitik weiter ansteigen wird. Die kommende Strategic Energy Review ist deshalb weniger als Instrument der politischen Steuerung einzustufen, sondern vielmehr als ein Mittel der politischen Kommunikation. Die Bereitschaft zu einer ernsthaften Überprüfung der bisherigen EU-Energie- und Klimapolitik ist demgegenüber erst dann zu erwarten, wenn die internationalen Verhandlungen über ein Post-Kyoto-Regime abgeschlossen sein werden, frühestens also Anfang 2010.

\section{Aktuelle Neuerscheinung}

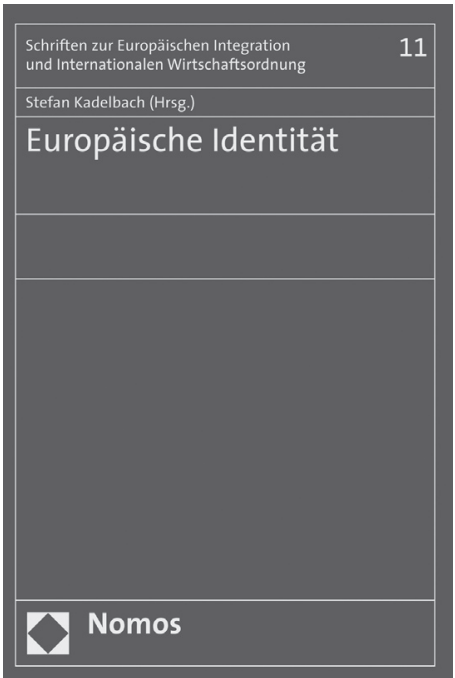

Europäische Identität

Herausgegeben von

Prof. Dr. Stefan Kadelbach

2008, 67 S., brosch., 19,- $€$,

ISBN 978-3-8329-3533-7

(Schriften zur Europäischen Integration und Internationalen Wirtschaftsordnung, $B d .11)$

Der Tagungsband enthält die Referate des 6. Walter Hallstein-Kolloquiums. Die einzelnen Beiträge analysieren aus interdisziplinärer Perspektive die Grundlagen und Facetten der europäischen Identität. 